\title{
Pulsed Laser Heating and Melting
}

\author{
David Sands \\ University of Hull \\ $U K$
}

\section{Introduction}

Modification of surfaces by laser heating has become a very important aspect of modern materials science. The author's own interests in laser processing have been involved in the main with laser processing of semiconductors, especially II-VI materials such as CdTe, but also amorphous silicon. Applications of laser processing are diverse and include, in addition to the selective recrystallisation of amorphous semiconductors, the welding of metals and other functional materials, such as plastic, case hardening in tool steels, and phase changes in optical data storage media. In addition there are numerous ideas under investigation in research laboratories around the world that have not yet become commercial applications and perhaps never will, but make use of the advantages and flexibility afforded by laser irradiation for both fundamental research into materials as well as small scale fabrication and technological innovation.

The range of laser types is truly staggering, but from the perspective of heat conduction it is possible to regard the laser simply as black box that provides a source of heat over a period of time that can range from femtoseconds to many tens of seconds, the latter effectively corresponding to continuous heating. The laser is therefore an incredibly versatile tool for effecting changes to the surfaces of materials, with the depth of material affected ranging from a few nanometres to several hundreds of microns, and possibly even millimetres. Strictly, a full discussion of surface modification by laser processing should include laser ablation and marking, but, interesting though the physics and technology undoubtedly are, attention will instead be restricted to the range of temperatures up to and beyond melting but excluding material removal by ablation as the loss of material from the surface represents for the purposes of this chapter a significant loss of energy which is then no longer available for heat conduction into the bulk.

Despite this apparent diversity in both the lasers and the possible processes, models of heat conduction due to laser irradiation share many common features. For pulse durations longer than a nanosecond or so thermal transport is essentially based on Fourier's law whilst for shorter pulses the models need to account for the separate contributions from both electrons and phonons. Geometry can also greatly simplify the modelling. Many processes typically involve moving a work-piece, or target, against a stationary beam (figure 1a), which can be either pulsed repetitively or continuous. The intensity of the beam may vary laterally or not (figure 1b), so a model of a moving work-piece might have to account for either a temporally varying intensity as the material encounters first the low-intensity leading edge, then the high-intensity middle, and finally the low-intensity trailing edge, or 
the cumulative effect of a number of pulses of equal intensity. In much of materials research the aim is to determine the effect of laser radiation on some material property and this kind of moving geometry can represent an unnecessary complication. The material is therefore heated statically for a limited time and the intensity is often, but not always, assumed to be constant over this time. These simplifications are often essential in order to make the problem mathematically tractable or in order to reduce the computation time for numerical models. A number of such models will be described, starting with analytical models of thermal transport.

(a)

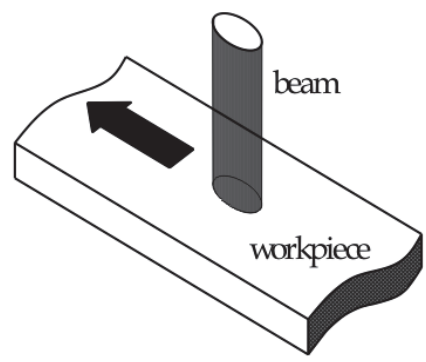

(b)

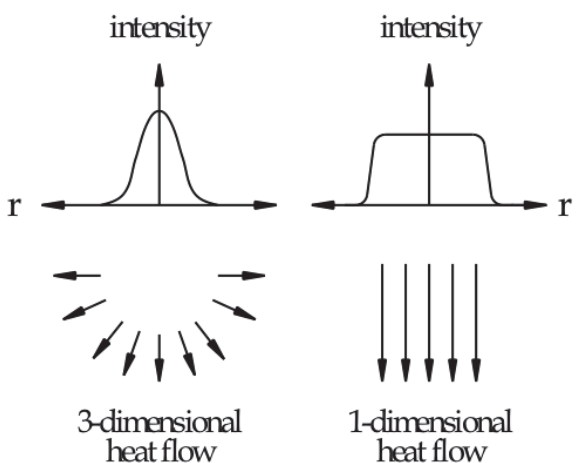

Fig. 1. Typically a workpiece, or target, is scanned relative to a stationary laser beam (a), which might have a non-uniform intensity profile across the beam radius, $r$, leading to 3dimensional heat flow or a "top hat" profile leading to 1-dimensional heat flow (b).

\section{Laser heating basics}

As indicated above, analytical models of laser processing represent a simplification of the real process, but they can nonetheless provide valuable insight into the response of the material. Attention is restricted in this section to pulses longer than a nanosecond, for which the energy of the lattice can be assumed to follow the laser pulse. As described by von Allmen (von Allmen \& Blatter, 1995) electrons which gain energy by absorbing a photon will relax back to the ground state by giving up their energy to the lattice within a few picoseconds, so at the time scales of interest here, which for convenience we shall call the long-pulse condition, the details of this process can be ignored and it can safely be assumed that any optical energy absorbed from the laser beam manifests itself as heat. This heat propagates through the material according to Fourier's law of heat conduction:

$$
\frac{d Q}{d t}=-k \nabla T
$$

The coefficient $k$ is known as the thermal conductivity and $\nabla$ is the gradient operator.

Fourier's law is empirical and essentially describes thermal diffusion, analogous to Fick's first law of diffusion. Heat is a strange concept and was thought at one stage by early thermodynamicists to represent the total energy contained in a body. This is not the case and in a thermodynamic sense heat represents an interaction; an exchange of energy not in 
the form of work that changes the total internal energy of the body. There is no sense in modern thermodynamics of the notion of the heat contained in a body, but in the present context the energy deposited within a material by laser irradiation manifests itself as heating, or a localised change in temperature above the ambient conditions, and it seems on the face of it to be a perfectly reasonable idea to think of this energy as a quantity of heat. Thermodynamics reserves the word enthalpy, denoted by the symbol $H$, for such a quantity and henceforth this term will be used to describe the quantity of energy deposited within the body. A small change in enthalpy, $\Delta H$, in a mass of material, $m$, causes a change in temperature, $\Delta T$, according to.

$$
\Delta H=m c_{p} \Delta T
$$

The quantity $c_{p}$ is the specific heat at constant pressure. In terms of unit volume, the mass is replaced by the density $\rho$ and

$$
\Delta H_{V}=\rho c_{p} \Delta T
$$

Equations (2) and (3) together represent the basis of models of long-pulse laser heating, but usually with some further mathematical development. Heat flows from hot to cold against the temperature gradient, as represented by the negative sign in eqn (1), and heat entering a small element of volume $\Delta \mathrm{V}$ must either flow out the other side or change the enthalpy of the volume element. Mathematically, this can be represented by the divergence operator

$$
\nabla \cdot \tilde{Q}=-\frac{d H_{V}}{d t}
$$

where $\tilde{Q}=d Q / d t$ is the rate of flow of heat. The negative sign is required because the divergence operator represents in effect the difference between the rate of heat flow out of a finite element and the rate of heat flow into it. A positive divergence therefore means a nett loss of heat within the element, which will cool as a result. A negative divergence, ie. more heat flowing into the element than out of it, is required for heating.

If, in addition, there is an extra source of energy, $S(z)$, in the form of absorbed optical radiation propagating in the $z$-direction normal to a surface in the $x-y$ plane, then this must contribute to the change in enthalpy and

$$
S(z)-\nabla \cdot \tilde{Q}=\frac{d H_{V}}{d t}
$$

Expanding the divergence term on the left,

$$
-\nabla \bullet \tilde{Q}=\nabla \bullet(k \nabla T)=k \nabla^{2} T+\nabla k \bullet \nabla T
$$

In Cartesian coordinates, and taking into account equations (3), (4) and (5)

$$
\frac{d T}{d t}=\frac{k}{\rho c_{p}}\left(\frac{\partial^{2} T}{\partial x^{2}}+\frac{\partial^{2} T}{\partial y^{2}}+\frac{\partial^{2} T}{\partial z^{2}}\right)+\frac{1}{\rho c_{p}}\left(\frac{\partial k}{\partial x} \frac{\partial T}{\partial x}+\frac{\partial k}{\partial y} \frac{\partial T}{\partial y}+\frac{\partial k}{\partial z} \frac{\partial T}{\partial z}\right)+\frac{S(z)}{\rho c_{p}}
$$


The source term in (7) can be derived from the laws of optics. If the intensity of the laser beam is $I_{0}$, in $\mathrm{Wm}^{-2}$, then an intensity, $I_{T}$, is transmitted into the surface, where

$$
I_{T}=I_{0}(1-R)
$$

Here $\mathrm{R}$ is the reflectivity, which can be calculated by well known methods for bulk materials or thin film systems using known data on the refractive index. Even though the energy density incident on the sample might be enormous compared with that used in normal optical experiments, for example a pulse of $1 \mathrm{~J} \mathrm{~cm}^{-2}$ of a nanosecond duration corresponds to a power density of $10^{9} \mathrm{Wcm}^{-2}$, significant non-linear effects do not occur in normal materials and the refractive index can be assumed to be unaffected by the laser pulse.

The optical intensity decays exponentially inside the material according to

$$
I(z)=I_{T} \exp (-\alpha z)
$$

where $\alpha$ is the optical absorption coefficient. Therefore

$$
S(z)=\alpha I(z)=\alpha I_{0}(1-R) \exp (-\alpha z)
$$

Analytical and numerical models of pulsed laser heating usually involve solving equation (7) subject to a source term of the form of (10). There have been far too many papers over the years to cite here, and too many different models of laser heating and melting under different conditions of laser pulse, beam profile, target geometry, ambient conditions, etc. to describe in detail. As has been described above, analytical models usually involve some simplifying assumptions that make the problem tractable, so their applicability is likewise limited, but they nonetheless can provide a valuable insight into the effect of different laser parameters as well as provide a point of reference for numerical calculations. Numerical calculations are in some sense much simpler than analytical models as they involve none of the mathematical development, but their implementation on a computer is central to their accuracy. If a numerical calculation fails to agree with a particular analytical model when run under the same conditions then more than likely it is the numerical calculation that is in error.

\section{Analytical solutions}

\subsection{Semi-infinite solid with surface absorption}

Surface absorption represents a limit of very small optical penetration, as occurs for example in excimer laser processing of semiconductors. The absorption depth of $\mathrm{UV} \mathrm{nm}$ radiation in silicon is less than $10 \mathrm{~nm}$. Although it varies slightly with the wavelength of the most common excimer lasers it can be assumed to be negligible compared with the thermal penetration depth. Table 1 compares the optical and thermal penetration in silicon and gallium arsenide, two semiconductors which have been the subject of much laser processing research over the years, calculated using room temperature thermal and optical properties at various wavelengths commonly used in laser processing.

It is evident from the data in table 1 that the assumption of surface absorption is justified for excimer laser processing in both semiconductors, even though the thermal penetration depth in GaAs is just over half that of silicon. However, for irradiation with a Q-switched $\mathrm{Nd}$ :YAG laser, the optical penetration depth in silicon is comparable to the thermal penetration and a different model is required. GaAs has a slightly larger band gap than silicon and will not absorb at all this wavelength at room temperature. 


\begin{tabular}{|c|c|c|c|c|c|c|}
\hline Laser & $\begin{array}{c}\text { Wavelength } \\
(\mathrm{nm})\end{array}$ & $\begin{array}{c}\text { Typical pulse } \\
\text { length } \\
\tau(\mathrm{ns})\end{array}$ & \multicolumn{2}{|c|}{$\begin{array}{c}\text { Thermal penetration } \\
\text { depth, }(\mathrm{D} \tau)^{1 / 2}(\mathrm{~nm})\end{array}$} & \multicolumn{2}{|c|}{$\begin{array}{c}\text { Optical penetration } \\
\text { depth, } \alpha^{-1}(\mathrm{~nm})\end{array}$} \\
\hline & 308 & 30 & 1660 & 973 & 6.8 & 12.8 \\
\hline XeCl excimer & 248 & 30 & 1660 & 973 & 5.5 & 4.8 \\
\hline KrF excimer & 192 & 30 & 1660 & 973 & 5.6 & 10.8 \\
\hline $\begin{array}{c}\text { ArF } \\
\text { excimer }\end{array}$ & 1060 & 6 & 743 & 435 & 1000 & sallium \\
\hline $\begin{array}{c}\text { Q-switched } \\
\text { Nd:YAG }\end{array}$ & silicon & $\begin{array}{c}\text { Gallium } \\
\text { arsenide }\end{array}$ \\
\hline
\end{tabular}

Table 1. The thermal and optical penetration into silicon and gallium arsenide calculated for commonly used pulsed lasers.

Assuming, then, surface absorption and temperature-independent thermo-physical properties such as conductivity, density and heat capacity, it is possible to solve the heat diffusion equations subject to boundary conditions which define the geometry of the sample. For a semiinfinite solid heated by a laser with a beam much larger in area than the depth affected, corresponding to $1-\mathrm{D}$ thermal diffusion as depicted in figure $1 \mathrm{~b}$, equation (7) becomes

$$
\frac{d T}{d t}=D \frac{\partial^{2} T}{\partial z^{2}}
$$

Here $k$ is the thermal conductivity and $D=\frac{k}{\rho c_{p}}$ the thermal diffusivity. Surface absorption implies

$$
\begin{gathered}
S(0)=\alpha I(0)=\alpha I_{0}(1-R) \\
S(z)=0, z>0
\end{gathered}
$$

Solution of the 1-D heat diffusion equation (11) yields the temperature, $T$, at a depth $z$ and time $t$ shorter than the laser pulse length, $\tau$, (Bechtel, 1975)

$$
T(z, t<\tau)=\frac{2 \alpha I_{0}(1-R)}{k}(D t)^{\frac{1}{2}} \operatorname{ierfc}\left[\frac{z}{2(D t)^{\frac{1}{2}}}\right]
$$

The integrated complementary error function is given by

$$
\operatorname{ierfc}(z)=\int_{z}^{\infty} \operatorname{erfc}(\xi) d \xi
$$

with

$$
\operatorname{erfc}(z)=1-\operatorname{erf}(z)=1-\frac{2}{\sqrt{\pi}} \int_{0}^{x} e^{-t^{2}} d t
$$


The surface $(z=0)$ temperature is given by,

$$
T(0, t<\tau)=\frac{2 \alpha I_{0}(1-R)}{k}(D t)^{\frac{1}{2}}\left(\frac{1}{\pi}\right)^{\frac{1}{2}}
$$

For times greater than the pulse duration, $\tau$, the temperature profile is given by a linear combination of two similar terms, one delayed with respect to the other. The difference between these terms is equivalent to a pulse of duration $\tau$ (figure 2).

$$
T(z, t>\tau)=\frac{2 \alpha I_{0}(1-R)}{k}\left\{(D t)^{\frac{1}{2}} i \operatorname{ierfc}\left[\frac{z}{2(D t)^{\frac{1}{2}}}\right]-[D(t-\tau)]^{\frac{1}{2}} i \operatorname{ierfc}\left[\frac{z}{2[D(t-\tau)]^{\frac{1}{2}}}\right]\right\}
$$

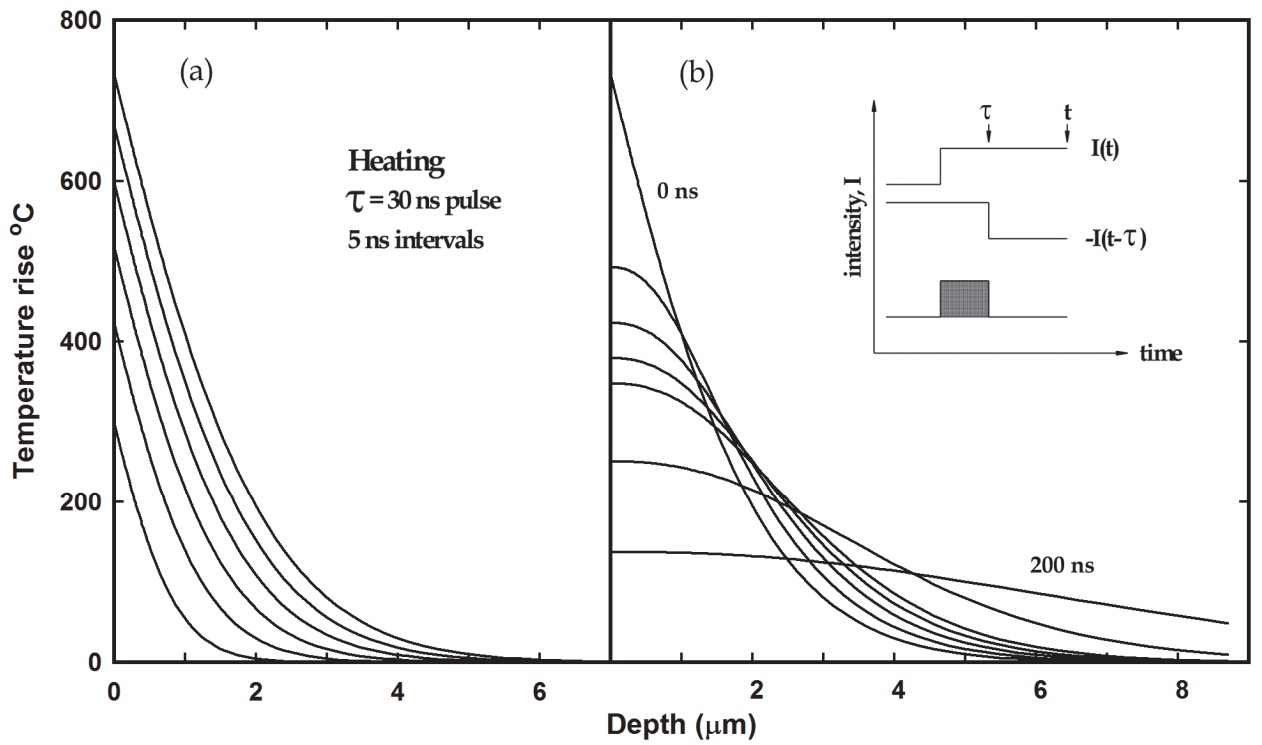

Fig. 2. Solution of equations (14) and (18) for a $30 \mathrm{~ns}$ pulse of energy density $400 \mathrm{~mJ} \mathrm{\textrm {cm } ^ { - 2 }}$ incident on crystalline silicon with a reflectivity of 0.56 . The heating curves (a) are calculated at 5 ns intervals up to the pulse duration and the cooling curves are calculated for 5, 10, 15, 20, 50 and $200 \mathrm{~ns}$ after the end of the laser pulse according to the scheme shown in the inset.

\subsection{Semi-infinite solid with optical penetration}

Complicated though these expressions appear at first sight, they are in fact simplified considerably by the assumption of surface absorption over optical penetration. For example, for a spatially uniform source incident on a semi-infinite slab, the closed solution to the heat transport equations with optical penetration, such as that given in Table 1 for Si heated by pulsed Nd:YAG, becomes (von Allmen \& Blatter, 1995) 


$$
T(z, t)=\frac{\alpha I_{0}(1-R)}{k}\left\{\begin{array}{l}
2(D t)^{\frac{1}{2}} i e r f c\left[\frac{z}{2(D t)^{\frac{1}{2}}}\right]-\frac{1}{\alpha} e^{-\alpha z} \\
+\frac{1}{2 \alpha} e^{\alpha^{2}(D t)} \times\left(e^{-\alpha z} \operatorname{erfc}\left[\alpha(D t)^{\frac{1}{2}}-\frac{z}{(D t)^{\frac{1}{2}}}\right]+e^{\alpha z} \operatorname{erfc}\left[\alpha(D t)^{\frac{1}{2}}+\frac{z}{(D t)^{\frac{1}{2}}}\right]\right.
\end{array}\right\}
$$

\subsection{Two layer heating with surface absorption}

The semi-infinite solid is a special case that is rarely found within the realm of high technology, where thin films of one kind or another are deposited on substrates. In truth such systems can be composed of many layers, but each additional layer adds complexity to the modelling. Nonetheless, treating the system as a thin film on a substrate, while perhaps not always strictly accurate, is better than treating it as a homogeneous body. ElAdawi et al (El-Adawi et al, 1995) have developed a two-layer of model of laser heating which makes many of the same assumptions as described above; surface absorption and temperature independent thermophysical properties, but solves the heat diffusion equation in each material and matches the solutions at the boundary. We want to find the temperature at a time $\mathrm{t}$ and position $z=z_{f}$ within a thin film of thickness $Z$, and the temperature at a position $z_{s}=z-Z$ within the substrate. If the thermal diffusivity of the film and substrate are $\alpha_{f}$ and $\alpha_{s}$ respectively then the parabolic diffusion equation in either material can be written as

$$
\left.\begin{array}{l}
\frac{\partial T_{f}\left(z_{f}, t\right)}{\partial t}=D_{f} \frac{\partial^{2} T_{f}\left(z_{f}, t\right)}{\partial z_{f}^{2}}, 0 \leq z_{f} \leq Z \\
\frac{\partial T_{s}\left(z_{s}, t\right)}{\partial t}=D_{s} \frac{\partial^{2} T_{s}\left(z_{s}, t\right)}{\partial z_{s}^{2}}, 0 \leq z_{s} \leq \infty
\end{array}\right\}
$$

These are solved by taking the Laplace transforms to yield a couple of similar differential equations which in general have exponential solutions. These can be transformed back once the coefficients have been found to give the temperatures within the film and substrate.

If $0 \leq n \leq \infty$ is an integer, then the following terms can be defined:

$$
\begin{aligned}
& \left.\begin{array}{l}
a_{n}=2 Z(1+n)-z_{f} \\
b_{n}=2 n Z+z_{f} \\
g_{n}=(1+2 n) Z+z_{s} \sqrt{\frac{D_{f}}{D_{s}}}
\end{array}\right\} \\
& L_{f}^{2}=4 D_{f} t
\end{aligned}
$$

The temperatures within the film and substrate are then given by 


$$
\begin{array}{r}
T_{f}\left(z_{f}, t\right)=\sum_{n=0}^{\infty} \frac{I_{0} A_{f}}{k_{f}} B^{n+1}\left[\frac{L_{f}}{\sqrt{\pi}} \exp \left(-\frac{a_{n}^{2}}{L_{f}^{2}}\right)-a_{n} \cdot \operatorname{erfc}\left(\frac{a_{n}}{L_{f}}\right)\right] \\
+\sum_{n=0}^{\infty} \frac{I_{0} A_{f}}{k_{f}} B^{n}\left[\frac{L_{f}}{\sqrt{\pi}} \exp \left(-\frac{b_{n}^{2}}{L_{f}^{2}}\right)-b_{n} \cdot \operatorname{erfc}\left(\frac{b_{n}}{L_{f}}\right)\right] \\
\left.T_{s}\left(z_{s}, t\right)=\sum_{n=0}^{\infty} \frac{2 I_{0} A_{f}}{k_{f}} \frac{B^{n}}{(1+\varepsilon)}\left[\frac{L_{f}}{\sqrt{\pi}} \exp \left(-\frac{g_{n}^{2}}{L_{f}}\right)-g_{n} \cdot \operatorname{erfc}\left(\frac{g_{n}}{L_{f}}\right)\right]\right\}
\end{array}
$$

Here $I_{0}$ is the laser flux, or power density, $A_{f}$ is the surface absorptance of the thin film material, $\mathrm{k}_{\mathrm{f}}$ is the thermal conductivity of the film and

$$
B=\frac{1-\varepsilon}{1+\varepsilon}<1
$$

It follows, therefore, that higher powers of $B$ rapidly become negligible as the index increases and in many cases the summation above can be curtailed for $n>10$. The parameter $\varepsilon$ is defined as

$$
\varepsilon=\frac{k_{s}}{k_{f}} \sqrt{\frac{D_{f}}{D_{s}}}
$$

Despite their apparent simplicity, at least in terms of the assumptions if not the final form of the temperature distribution, these analytical models can be very useful in laser processing. In particular, El-Adawi's two-layer model reduces to the analytical solution for a semiinfinite solid with surface absorption (equation 14) if both the film and the substrate are given the same thermal properties. This means that one model will provide estimates of the temperature profile under a variety of circumstances. The author has conducted laser processing experiments on a range of semiconductor materials, such as $\mathrm{Si}$, CdTe and other II-VI materials, $\mathrm{GaAs}$ and $\mathrm{SiC}$, and remarkably in all cases the onset of surface melting is observed to occur at an laser irradiance for which the surface temperature calculated by this model lies at, or very close to, the melting temperature of the material. Moreover, by the simple expedient of subtracting a second expression, as in equation (18) and illustrated in the inset of figure $2 b$, the temperature profile during the laser pulse and after, during cooling, can also be calculated. El-Adawi's two-layer model has thus been used to analyse time-dependent reflectivity in laser irradiated thin films of ZnS on Si (Hoyland et al, 1999), calculate diffusion during the laser pulse in GaAs (Sonkusare et al, 2005) and CdMnTe (Sands et al, 2000), and examine the laser annealing of ion implantation induced defects in CdTe (Sands \& Howari, 2005).

\section{Analytical models of melting}

Typically, analytical models tend to treat simple structures like a semi-infinite solid or a slab. Equation (22) shows how complicated solutions can be for even a simple system comprising only two layers, and if a third were to be added in the form of a time-dependent molten layer, the mathematics involved would become very complicated. One of the earliest 
models of melting considered the case of a slab either thermally insulated at the rear or thermally connected to some heat sink with a predefined thermal transport coefficient. Melting times either less than the transit time (El-Adawi, 1986) or greater than the transit time (El-Adawi \& Shalaby, 1986) were considered separately. The transit time in this instance refers to the time required for temperature at the rear interface to increase above ambient, ie. when heat reaches the rear interface, located a distance $l$ from the front surface, and has a clear mathematical definition.

The detail of El-Adawi's treatment will not be reproduced here as the mathematics, while not especially challenging in its complexity, is somewhat involved and the results are of limited applicability. Partly this is due to the nature of the assumptions, but it is also a limitation of analytical models. As with the simple heating models described above, ElAdawi assumed that heat flow is one-dimensional, that the optical radiation is entirely absorbed at the surface, and that the thermal properties remain temperature independent. The problem then reduces to solving the heat balance equation at the melt front,

$$
I_{0} A(1-R)+k \frac{d T}{d z}=\rho_{s} L \frac{d Z}{d t}
$$

Here $Z$ represents the location of the melt front and any value of $Z \leq z \leq l$ corresponds to solid material. The term on the right hand side represents the rate at which latent heat is absorbed as the melt front moves and the quantity $L$ is the latent heat of fusion. Notice that optical absorption is assumed to occur at the liquid-solid interface, which is unphysical if the melt front has penetrated more than a few nanometres into the material. The reason for this is that El-Adawi fixed the temperature at the front surface after the onset of melting at the temperature of the phase change, $T_{m}$. Strictly, there would be no heat flow from the absorbing surface to the phase change boundary as both would be at the same temperature, so in effect El-Adawi made a physically unrealistic assumption that molten material is effectively evaporated away leaving only the liquid-solid interface as the surface which absorbs incoming radiation.

El-Adawi derived quadratic equations in both $Z$ and $d Z / d t$ respectively, the coefficients of which are themselves functions of the thermophysical and laser parameters. Computer solution of these quadratics yields all necessary information about the position of the melt front and El-Adawi was able to draw the following conclusions. For times greater than the critical time for melting but less than the transit time the rate of melting increases initially but then attains a constant value. For times greater than the critical time for melting but longer than the transit time, both $Z$ and $d Z / d t$ increase almost exponentially, but at rates depending on the value of $h$, the thermal coupling of the rear surface to the environment. This can be interpreted in terms of thermal pile-up at the rear surface; as the temperature at the rear of the slab increases this reduces the temperature gradient within the remaining solid, thereby reducing the flow of heat away from the melt front so that the rate at which material melts increases with time.

The method adopted by El-Adawi typifies mathematical approaches to melting in as much as simplifying assumptions and boundary conditions are required to render the problem tractable. In truth one could probably fill an entire chapter on analytical approaches to melting, but there is little to be gained from such an exercise. Each analytical model is limited not only by the assumptions used at the outset but also by the sort of information that can be calculated. In the case of El-Adawi's model above, the temperature profile within 
the molten region is entirely unknown and cannot be known as it doesn't feature in the formulation of the model. The models therefore apply to specific circumstances of laser processing, but have the advantage that they provide approximate solutions that may be computed relatively easily compared with numerical solutions. For example, El-Adawi's model of melting for times less than the transit time is equivalent to treating the material as a semi-infinite slab as the heat has not penetrated to the rear surface. Other authors have treated the semi-infinite slab explicitly. Xie and Kar (Xie \& Kar, 1997) solve the parabolic heat diffusion equation within the liquid and solid regions separately and use similar heat balance equations. That is, the liquid and solid form a coupled system defined by a set of equations like (20) with $Z$ again locating the melt front rather than an interface between two different materials. The heat balance equation at the interface between the liquid and solid becomes

$$
k_{l} \frac{\partial T_{l}(z, t)}{\partial z}=k_{s} \frac{\partial T_{s}(z, t)}{\partial z}-\rho_{s} L \frac{d Z(t)}{d t}
$$

At the surface the heat balance is defined by

$$
I_{0} A(1-R)+k_{l} \frac{\partial T(0, t)}{\partial z}=0
$$

The solution proceeds by assuming a temperature within the liquid layer of the form

$$
T_{l}(z, t)=T_{m}-\frac{A I}{k_{l}}[z-Z(t)]+\psi(t)\left[z^{2}-Z(t)^{2}\right]
$$

The heat balance equation at $z=0$ then determines $\psi(t)$. Similarly the temperature in the solid is assumed to be given by

$$
T_{s}(z, t)=T_{m}-\left(T_{m}-T_{o}\right)[1-\exp (-b(t)[z-Z(t)])]
$$

The boundary conditions at $z=Z(t)$ then determine $b(t)$. Some further mathematical manipulation is necessary before arriving at a closed form which is capable of being computed. Comparison with experimental data on the melt depth as a function of time shows that this model is a reasonable, if imperfect, approximation that works quite well for some metals but less so for others.

Other models attempt to improve on the simplifying assumption by incorporating, for example, a temperature dependent absorption coefficient as well as the temporal variation of the pulse energy (Abd El-Ghany, 2001; El-Nicklawy et al, 2000). These are some of the simplest models; 1-D heat flow after a single pulse incident on a homogeneous solid target with surface absorption. In processes such as laser welding the workpiece might be scanned across a fixed laser beam (Shahzade et al, 2010), which in turn might well be Gaussian in profile (figure 1) and focussed to a small spot. In addition, the much longer exposure of the surface to laser irradiation leads to much deeper melting and the possibility of convection currents within the molten material (Shuja et al, 2011). Such processes can be treated analytically (Dowden, 2009), but the models are too complicated to do anything more than mention here. Moreover, the models described here are heating models in as much as they deal with the system under the influence of laser irradiation. When the irradiation source is 
removed and the system begins to cool, the problem then is to decide under what conditions the material begins to solidify. This is by no means trivial, as melting and solidification appear to be asymmetric processes; whilst liquids can quite readily be cooled below the normal freezing point the converse is not true and materials tend to melt once the melting point is attained.

Models of melting are, in principle at least, much simpler than models of solidification, but the dynamics of solidification are just as important, if not more so, than the dynamics of melting because it is upon solidification that the characteristic microstructure of laser processed materials appears. One of the attractions of short pulse laser annealing is the effect on the microstructure, for example converting amorphous silicon to large-grained polycrystalline silicon. However, understanding how such microstructure develops is impossible without some appreciation of the mechanisms by which solid nuclei are formed from the liquid state and develop to become the recrystallised material. Classical nucleation theory $(\mathrm{Wu}, 1997)$ posits the existence of one or more stable nuclei from which the solid grows. The radius of a stable nucleus decreases as the temperature falls below the equilibrium melt temperature, so this theory favours undercooling in the liquid. In like manner, though the theory is different, the kinetic theory of solidification (Chalmers and Jackson, 1956; Cahoon, 2003) also requires undercooling. The kinetic theory is an atomistic model of solidification at an interface and holds that solidification and melting are described by different activation energies. At the equilibrium melt temperature, $T_{m}$, the rates of solidification and melting are equal and the liquid and solid phases co-exist, but at temperatures exceeding $T_{m}$ the rate of melting exceeds that of solidification and the material melts. At temperatures below $T_{m}$ the rate of solidification exceeds that of melting and the material solidifies. However, the nett rate of solidification is given by the difference between the two rates and increases as the temperature decreases. The model lends itself to laser processing not only because the transient nature of heating and cooling leads to very high interface velocities, which in turn implies undercooling at the interface, but also because the common theory of heat conduction, that is, Fourier's law, across the liquid-solid interface implies it.

A common feature of the analytical models described above is the assumption that the interface is a plane boundary between solid and liquid that stores no heat. The idea of the interface as a plane arises from Fourier's law (equation 1) in conjunction with coexistence, the idea that liquid and solid phases co-exist together at the melt temperature. It follows that if a region exists between the liquid and solid at a uniform temperature then no heat can be conducted across it. Therefore such a region cannot exist and the boundary between the liquid and solid must be abrupt. An abrupt boundary implies an atomistic crystallization model; the solid can only grow as atoms within the liquid make the transition at the interface to the solid, which is of course the basis of the kinetic model. However, there has been growing recognition in recent years that this assumption might be wanting, especially in the field of laser processing where sometimes the melt-depth is only a few nanometres in extent. This opens the way to consideration of other recrystallisation mechanisms.

One possibility is transient nucleation (Shneidman, 1995; Shneidman and Weinberg, 1996), which takes into account the rate of cooling on the rate of nucleation. Most of Shneidman's work is concerned with nucleation itself rather than the details of heat flow during crystallisation, but Shneidman has developed an analytical model applicable to the solidification of a thin film of silicon following pulsed laser radiation (Shneidman, 1996). As 
with most analytical models, however, it is limited by the assumptions underlying it, and if details of the evolution of the microstructure in laser melted materials are required, this is much better done numerically. We shall return to the topic of the liquid-solid interface and the mechanism of re-crystallization after describing numerical models of heat conduction.

\section{Numerical methods in heat transfer}

Equations (1), (3) and (11), which form the basis of the analytical models described above, can also be solved numerically using a forward time step, finite difference method. That is, the solid target under consideration is divided into small elements of width $\delta z$, with element 1 being located at the irradiated surface. The energy deposited into this surface from the laser in a small interval of time, $\delta t$, is, in the case of surface absorption,

$$
\delta E=\alpha I_{0}(1-R) \delta t
$$

and

$$
\delta E=S(z) \delta t=\alpha I_{0}(1-R) \exp (-\alpha z) \cdot \delta t
$$

in the case of optical penetration. If the adjacent element is at a mean temperature $T_{2}$, assumed to be constant across the element, the heat flowing out of the first element within this time interval is

$$
\delta Q_{12}=-k \frac{\left(T_{2}-T_{1}\right)}{\delta z} . \delta t
$$

The enthalpy change in element 1 is therefore

$$
\delta H=\delta E-\delta Q_{12}=(\rho \delta z) c_{p} \delta T_{1}
$$

In this manner the temperature rise in element $1, \delta T_{1}$, can be calculated. The heat flowing out of element 1 flows into element 2 . Together with any optical power absorbed directly within the element as well as the heat flowing out of element 2 and into 3, this allows the temperature rise in element 2 to be calculated. This process continues until an element at the ambient temperature is reached, and conduction stops. In practice it might be necessary to specify some minimum value of temperature below which it is assumed that heat conduction does not occur because it is a feature of Fourier's law that the temperature distribution is exponential and in principle very small temperatures could be calculated. However the matter is decided in practice, once heat conduction ceases the time is stepped on by an amount $\delta t$ and the cycle of calculations is repeated again. In this way the temperature at the end of the pulse can be calculated or, if the incoming energy is set to zero, the calculation can be extended beyond the duration of the laser pulse and the system cooled.

This is the essence of the method and the origin of the name "forward time step, finite difference", but in practice calculations are often done differently because the method is slow; the space and time intervals are not independent and the total number of calculations is usually very large, especially if a high degree of spatial accuracy is required. However, this is the author's preferred method of performing numerical calculations for reasons which will become apparent. The calculation is usually stable if 


$$
\delta z^{2}>2 D \cdot \delta t
$$

but the stability can be checked empirically simply by reducing $\delta t$ at a fixed value of $\delta z$ until the outcome of the calculation is no longer affected by the choice of parameters.

In order to overcome the inherent slowness of this technique, which involves explicit calculations of heat fluxes, alternative schemes based on the parabolic heat diffusion equation are commonly reported in the literature. It is relatively straightforward to show that between three sequential elements, say $j-1, j$ and $j+1$, with temperature gradients

$$
\begin{aligned}
& \frac{d T_{j-1, j}}{d z} \approx \frac{\left(T_{j}-T_{j-1}\right)}{\delta z} . \\
& \frac{d T_{j, j+1}}{d z} \approx \frac{\left(T_{j+1}-T_{j}\right)}{\delta z} .
\end{aligned}
$$

the second differential is given by

$$
\frac{d^{2} T}{d z^{2}} \approx \frac{\left(T_{j+1}-2 T_{j}+T_{j-1}\right)}{\delta z^{2}} .
$$

Hence the parabolic heat diffusion equation becomes

$$
\frac{d T_{j}}{d t} \approx \frac{\delta T_{j}}{\delta t}=D \frac{\left(T_{j+1}-2 T_{j}+T_{j-1}\right)}{\delta z^{2}} \cdot+\frac{1}{\rho c_{p}} \frac{\left(k_{j+1}-k_{j-1}\right)}{2 \delta z} \cdot \frac{\left(T_{j+1}-T_{j-1}\right)}{2 \delta z}
$$

with appropriate source terms of the form of equation (31) for any optical radiation absorbed within the element. Thus if the temperature of any three adjacent elements is known at any given time the temperature of the middle element can be calculated at some time $\delta t$ in the future without calculating the heat fluxes explicitly. This particular scheme is known as the forward-time, central-space (FTCS) method, but there are in fact several different schemes and a great deal of mathematical and computational research has been conducted to find the fastest and most efficient methods of numerical integration of the parabolic heat diffusion equation (Silva et al, 2008; Smith, 1965).

The difficulty with this equation, and the reason why the author prefers the more explicit, but slower method, lies in the second term, which takes into account variations in thermal conductivity with depth. Such changes can arise as a result of using temperature-dependent thermo-physical properties or across a boundary between two different materials, including a phase-change. However, Fourier's law itself is not well defined for heat flow across a junction, as the following illustrates. Mathematically, Fourier's law is an abstraction that describes heat flow across a temperature gradient at a point in space. A point thus defined has no spatial extension and strictly the problem of an interface, which can be assumed to be a 2-dimensional surface, does not arise in the calculus of heat flow. Besides, in simple problems the parabolic equation can be solved on both sides of the boundary, as was described earlier in El-Adawi's two-layer model, but in discrete models of heat flow, the location of an interface relative to the centre of an element assumes some importance. Within the central-space scheme the interface coincides with the boundary between two elements, say $j$ and $j+1$ with thermal conductivities $k_{j}$ and $k_{j+1}$ and temperatures $T_{j}$ and $T_{j+1}$. 
The thermal gradient can be defined according to equation (35), but the expression for the rate of flow of heat requires a thermal conductivity which changes between the elements. Which conductivity do we use; $k_{j}, k_{j+1}$ or some combination of the two?

This difficulty can be resolved by recognising that the temperatures of the elements represent averages over the whole element and therefore represent points that lie on a smooth curve. The interface between each element therefore lies at a well defined temperature and the heat flow can be written in terms of this temperature, $T_{i}$, as

$$
\begin{gathered}
\frac{d Q_{j+1, j}}{d t}=\frac{d Q_{j, i}}{d t}=-k_{j} \frac{T_{i}-T_{j}}{\delta z / 2} \\
\frac{d Q_{j+1, j}}{d t}=\frac{d Q_{i, j+1}}{d t}=-k_{j+1} \frac{T_{j+1}-T_{i}}{\delta z / 2}
\end{gathered}
$$

Solving for $T_{i}$ in terms of $T_{j}$ and $T_{j+1}$, it can be shown that

$$
T_{i}=\frac{k_{j} T_{j}+k_{j+1} T_{j+1}}{k_{j}+k_{j+1}}
$$

Substituting back into either of equations (38a) or (38b) yields

$$
\frac{d Q_{j, j+1}}{d t}=-2 \cdot \frac{k_{j} k_{j+1}}{k_{j}+k_{j+1}}\left[\frac{T_{j+1}-T_{j}}{\delta z}\right]
$$

The correct thermal conductivity in the discrete central-space method is therefore a composite of the separate conductivities of the adjacent cells. This is in fact entirely general, and applies even if the interface between the two cells does not coincide with the interface between two different materials. For example, if the two conductivities, $k_{j}$ and $k_{j+1}$ are identical the effective conductivity reduces simply to the conductivity $k_{j}=k_{j+1}$. If, however, the two cells, $j$ and $j+1$, comprise different materials such that the thermal conductivity of one vastly exceeds the other the effective conductivity reduces to twice the small conductivity and the heat flow is limited by the most thermally resistive material. For small changes in $k$ such that $k_{j}=k_{j-1}+\delta k$ and $k_{j+1}=k_{j}+\delta k=k_{j-1}+2 \delta k$, the difference in heat flow between the three elements can be written in terms of $k_{j-1}$ and $\delta k$. After some manipulation it can be shown that

$$
\nabla \bullet\left[\frac{d Q}{d t}\right] \approx \frac{k_{j-1}}{\delta z^{2}}\left[T_{j+1}-2 T_{j}-T_{j-1}\right]+\frac{\delta k}{\delta z} \cdot \frac{\delta T}{\delta z}
$$

with

$$
T_{j+1}-T_{j} \approx T_{j}-T_{j-1}=\delta T
$$

This is equivalent to equation (6) in one dimension. If, however, the change in thermal conductivity arises from a change in material such that $k_{j+1}=k_{j}+\delta k$ and $k_{j}=k_{j-1}$, and $\delta k$ need not be small in relation to $k_{j}$, then it can be shown that 


$$
\nabla \bullet\left[\frac{d Q}{d t}\right] \approx \frac{k_{j-1}}{\delta z^{2}}\left[T_{j+1}-2 T_{j}-T_{j-1}\right]+\frac{k_{j-1}}{\left(2 k_{j-1}+\delta k\right)} \frac{\delta k}{\delta z} \cdot \frac{\left(T_{j+1}-T_{j}\right)}{\delta z}
$$

We can consider two limiting cases. First, if $k_{j-1}>>k_{j+1}$, such that $\delta k \approx-k_{j-1}$ then

$$
\frac{k_{j-1}}{2 k_{j-1}+\delta k} \approx \frac{k_{j-1}}{k_{j-1}}=1
$$

In this case equation (43) approximates to equation (37). Secondly, if $k_{j-1}<<k_{j+1}$, such that $\delta k \approx k_{j+1}$ then

$$
\frac{k_{j-1}}{2 k_{j-1}+\delta k} \approx \frac{k_{j-1}}{k_{j+1}}<<1
$$

In this case the contribution from the second term in (43) is very small, but more importantly, equation (43) is shown not to be equivalent to (37). Likewise, if we choose some intermediate value, say $k_{j-1}=2 k_{j+1}$ or conversely $2 k_{j-1}=k_{j+1}$ this term becomes respectively $2 / 3$ or $1 / 3$. The precise value of this ratio will depend on the relative magnitudes of $k_{j-1}$ and $k_{j+1}$, but we see that in general equation (43) is not numerically equivalent to (37). The difference might only be small, but the cumulative effect of even small changes integrated over the duration of the laser pulse can turn out to be significant. For this reason the author's own preference for numerical solution of the heat diffusion equation involves explicit calculation of the heat fluxes into and out of an element according to equation (40) and explicit calculation of the temperature change within the element according to equation (3). As described, the method is slow, but the results are sure.

\subsection{Melting within numerical models}

The advantage of numerical modelling over analytical solutions of the heat diffusion equation is the flexibility in terms of the number of layers within the sample, the use of temperature dependent thermo-physical and optical properties as well as the temporal profile of the laser pulse. This advantage should, in principle, extend to treatments of melting, but self-consistent numerical models of melting and recrystallisation present considerable difficulty. Chalmers and Jackson's kinetic theory of solidification described previously implies that a fast rate of solidification, as found, for example, in nano-second laser processing, should be accompanied by significant undercooling of the liquid-solid interface. However, tying the rate of cooling to the rate of solidification within a numerical model presents considerable difficulties. Moreover, it might not be necessary.

In early work on laser melting of silicon it was postulated that an interface velocity of approximately $15 \mathrm{~ms}^{-1}$ is required to amorphise silicon. Amorphous silicon is known to have a melting point some $200^{\circ} \mathrm{C}$ below the melting point of crystalline silicon so it was assumed that in order to form amorphous silicon from the melt the interface must cool by at least this amount, which requires in turn such high interfacial velocities. By implication, however, the converse would appear to be necessary; that high rates of melting should be accompanied by overheating, yet the evidence for the latter is scant. Indeed, extensive modelling work in the 1980s on silicon (Wood \& Jellison, 1984), and GaAs (Lowndes, 1984) showed that very 
high interface velocities arise from the rate of heating supplied by the laser rather than any change in the temperature of the interface. These authors held the liquid-solid interface at the equilibrium melt temperature and calculated curves of the kind shown in figure $3 a$. Differentiation of the melt front position with respect to time (figure $3 b$ ) shows that the velocity during melting can exceed $20 \mathrm{~ms}^{-1}$ and during solidification can reach as high as 6 $\mathrm{ms}^{-1}$, settling at $3 \mathrm{~ms}^{-1}$. The fact of such large interface velocities does not, of itself, invalidate the notion of undercooling but it does mean that undercooling need not be a pre-requisite for, or indeed a consequence of, a high melt front velocity.

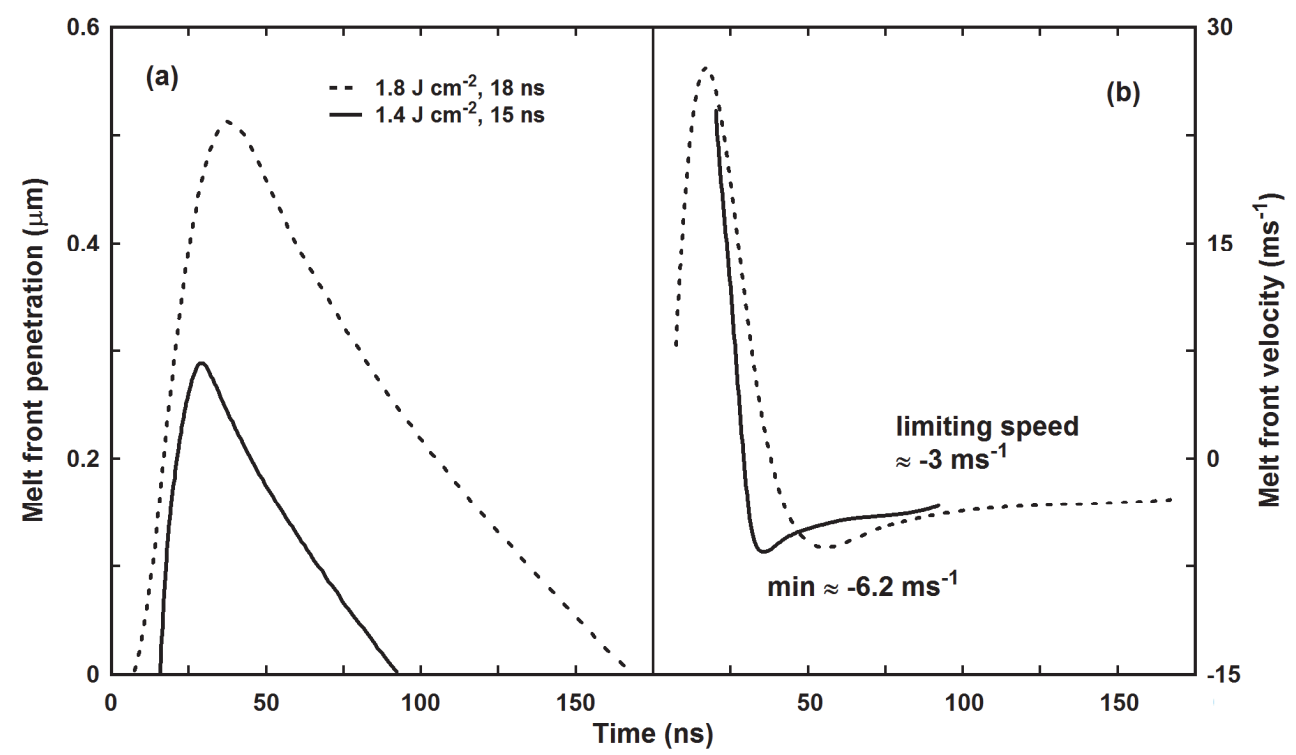

Fig. 3. Typical curves of the melt front penetration (a) taken from figures 4 and 6 of Wood and Jellison (1984) and the corresponding interface velocity (b).

If undercooling is not necessary for large interface velocities then the requirement that the interface be sharp, which is required by both the kinetic model of solidification and Fourier's law, might also be unnecessary. Various attempts have been made over the years to define an interface layer but the problem of ascribing a temperature to it is not trivial. The essential difficulty is that we have no knowledge of the thermal properties of materials in this condition, nor indeed a fully satisfactory theory of melting and solidification. One idea that has gained a lot of ground in recent years is the "phase field", a quantity, denoted by $\phi$, constructed within the theory of non-equilibrium thermodynamics that has the properties of a field but takes a value of either 0 or 1 for solid and liquid phases respectively and $0<\phi<1$ for the interphase region (Qin \& Bhadeshia, 2010; Sekerka, 2004). In essence, gradients within the thermodynamic quantities drive the process of crystallisation.

The phase field method was originally proposed for equilibrium solidification and has been very successful in predicting the large scale structure, such as the growth of dendrites, often seen in such systems. It has also been applied to rapid solidification (Kim \& Kim, 2001), including excimer laser processing of silicon (La Magna, 2004; Shih et al, 2006; Steinbach \& 
Apel, 2007). Despite its success in replicating many experimentally observed features in solidification (see for example, Pusztai, 2008, and references therein) and the phase field itself is not necessarily associated with any physical property of the interface (Qin \& Bhadeshia, 2010). Moreover, even though it can be adapted to apply to the numerical solution of the 1-D heat diffusion equation, it is essentially a method for looking at 2-D structures such as dendrites and is not well suited to planar interfaces. For example, in the work of Shih et al mentioned above, it was necessary to introduce a spherical droplet within the solid in order to initiate melting.

The author's own approach to this problem is to question the validity of Fourier's law in the domain of melting (Sands, 2007). It is necessary to state that either Fourier's law is invalid or a liquid and solid cannot co-exist at exactly the same temperature because the two concepts are mutually exclusive. Coexistence at the equilibrium melt temperature is, of course, a macroscopic idea that might, or might not apply at the microscopic level. It is difficult to imagine an experiment with sufficient resolution to measure the temperature either side of an interface, but if even a small difference exists it is sufficient for heat to flow according to Fourier's law. If no difference exists heat cannot flow across the interface. Of course, we know in practice that heat must flow in order to supply, or conduct away, the latent heat. This tension between the microscopic and macroscopic domains also applies to the model of the interface. The idea of a plane sharp interface that stores no heat arises in essence from mathematical models in which the heat diffusion equation is solved on either wide of the interface and the solutions matched. By definition, heat flowing out of one side flows into the other and the interface does nothing more than mark the point at which the phase changes. However, the idea of a fuzzy interface, as represented for example in phase field models, implies that interfaces do not behave like this at the microscopic level. More fundamental, however, is the question of whether a formulation of heat flow in terms of temperature or enthalpy per unit volume, $H_{v}$, is the more fundamental.

The parabolic heat diffusion equation arises from equations (1) and (4), with equation (3) being used to convert the rate of change of volumetric enthalpy to a rate of change of temperature. However, equation (3) can also be used to convert equation (1) to an expression for heat flow in terms of $H_{v}$, which now resembles Fick's first law of diffusion. Application of continuity, as expressed by equation (4), now leads to a parabolic equation in $H_{v}$ rather than $T$. Both forms of heat diffusion are mathematically valid, but they do not lead to the same outcome except in the case of a homogeneous material heated below the melting point. Whichever is the primary variable in the parabolic equation becomes continuous; temperature in one case, volumetric enthalpy in the other. Experience would seem to suggest that temperature is the more fundamental variable as thermal equilibrium between two different materials is expressed in terms of the equality of temperature rather than volumetric enthalpy, and indeed this is a weakness of the enthalpy formulation, but we have already seen in the derivation of equation (40) how the mathematical form of Fourier's law breaks down in numerical computation of heat flow across a junction. On the other hand, expressing the heat flow in terms of Fick's law of diffusion would seem to bring the idea of thermal diffusion in line with a host of other diffusion phenomena, thereby seeming to make this a more fundamental formulation. Moreover, it leads naturally to a diffuse model of the interface.

The width of the diffuse interface generated by the enthalpy model is much greater than the width of the liquid-solid interface observed in phase field models, but this is not in itself a difficulty. Nor does it imply that the normally accepted idea of coexistence is invalid, but it 
does require a different model of melting and solidification. The details are discussed by Sands (2007), but the ideas can be summarised as follows. Molecular dynamics simulations suggest that beyond a certain degree of disorder a defective solid changes abruptly into a liquid, so a continuous enthalpy can be interpreted as a mixture of liquid and solid regions co-existing together in a ratio that gives the average enthalpy of the mixture. However, coexistence is probably dynamic as the material fluctuates between solid and liquid forms.

The latter is more than just a fanciful idea. The classical theory of solidification is based on the idea of forming a stable nucleus of solid material at some temperature below the equilibrium melt temperature, but in fact such a model is not supported by the experimental evidence. It can be shown (Sands, 2007) that typically only $11 \%$ of the latent heat released during the formation of a solid nucleus is expended in the form of work done in forming the nucleus, meaning that the remainder must go into heating up the solid nucleus and the surrounding material. Indeed, it is a common observation in electrostatic levitation experiments (Sung et al, 2003) that once an undercooled melt begins to solidify the temperature rises to the equilibrium melt temperature. The phenomenon is known as recalescence. There is no requirement for solid nuclei to be stable under these conditions. Indeed, if a solid nucleus does in fact heat up its local environment then by implication the formation of a liquid nucleus within a solid matrix must cool the surrounding material. There is thus the possibility of large fluctuations in the temperature field, which could in turn trigger fluctuations between phases.

The advantage of the enthalpy formulation is that it is intuitive. The idea that an interface must achieve a certain velocity before amorphous solids can be formed is, in this author's view, no longer tenable. First, we have shown that computation using a fixed temperature lead to significant non-zero interface velocities, thereby breaking the link with undercooling. Secondly, the phenomenon of recalescence would tend to raise the temperature of the interface. Thirdly, it is well known that glassy metals are produced by rapid cooling which essentially freezes in the disorder associated with the liquid state. Although amorphous silicon is not a glass it is characterised by a similar disorder and it is not immediately clear why a similar mechanism cannot be responsible for its formation. The enthalpy formulation allows for this because thermal transport is determined essentially by the laser parameters; if the material does not crystallise before too much heat is lost amorphous silicon forms, but if crystallisation does occur and the rate of release of latent heat is faster than the rate at which heat is transported away then recalescence occurs. Whether crystallisation occurs or not is determined essentially by probability. Based on the author's work (Sands, 2007) nucleation in silicon would seem to require something in the region of 8-10 ns to initiate and amorphous silicon is formed because the system heats and cools within that timescale.

The weakness of the enthalpy formulation is undoubtedly its lack of self consistency, as it is necessary to switch to a temperature formulation at the interface between different materials. Indeed, it might be possible to treat the above ideas within a temperature formulation, because a model of the phase transition which accounts for recalescence could, in principle, be treated using Fourier's law. Unfortunately, such a general model has not yet been formulated and it is not clear to the author even that such ideas have been widely accepted. The difficulty lies in finding a simple formulation for the change in temperature associated with a transition from liquid to solid and vice versa, but if the microscopic variations in temperature could be formulated then large variations would exist across and within the interfacial layer and the resulting flow of heat would be quite complex. Finding a formulation of melting that is physically sensible and can be treated self consistently within 
an appropriate model of heat conduction is one of the most interesting problems in pulsed laser heating and melting.

\section{Short pulse heating}

The final topic of this chapter concerns heating using laser pulses of much shorter duration than the nanosecond pulses typical of excimer lasers and Q-switched YAG lasers. Lasers capable of delivering sub-picosecond pulses are now routinely used in materials processing, especially for ablation and marking, and a pre-requisite for modelling such processes is an accurate model of thermal transport, which is quite different from the mechanisms considered above. All the models of heating discussed above are predicated on the assumption that the temperature of the lattice follows the laser pulse, but with subpicosecond pulses that is no longer true; the electron and lattice temperatures have to be considered separately.

Models of short pulse heating have been around since the late 1980s, but papers continue to be published. One of the problems is the lack of data for different materials. Data for Ni and $\mathrm{Au}$ has been known for some time (Yilbas \& Shuja, 2000) and experiments on these materials continue to be performed (Chen, 2005), but data on silicon is more recent (Lee, 2005). The mechanisms of energy transport in semiconductors are also different from those in metals, where a two-temperature model is often invoked to explain ultra-fast heating (Yilbas \& Shuja, 1999). Photons are absorbed by electrons, which have a non-equilibrium energy distribution as a result. Electron-electron collisions establish thermal equilibrium within femtoseconds and electron-phonon (lattice vibrations) interactions transfer the energy from the electrons to the lattice in timescales of the order of picoseconds. A lot of theoretical papers are concerned with the nature of the electron-electron collisions (electron kinetic theory) and there is a suggestion (Rethfield et al, 2002b) that in fact the two-temperature model is inappropriate under conditions of weak excitation, as it takes longer than a picosecond for the electrons to establish an equilibrium distribution. On the other hand, for very strong excitation the electrons behave as if they follow a well defined Fermi-Dirac distribution and a temperature can be defined. The temperatures of the lattice and the electron distribution are then linked (Rethfield et al, 2002) by;

$$
c_{p} \frac{\partial T_{p}}{d t}=-c_{e} \frac{\partial T_{e}}{d t}=\alpha\left(T_{e}-T_{p}\right)
$$

The subscripts $p$ and $e$ refer to phonons (lattice) and electrons respectively, $c_{p}$ and $c_{e}$ are the respective heat capacities and $\alpha$ is the energy exchange rate between phonons and electrons. For semiconductors, however, three temperatures are needed to account for thermal transport (Lee, 2005); those of the electrons, the optical mode phonons and the acoustic mode phonons. Two-temperature models of semiconductor heating can be found (Medvedev \& Rethfeld, 2010), but this is only valid if thermal transport is neglected. That is, if the semiconductor is thin enough to be heated uniformly without thermal conduction. Otherwise, a three-stage process ought to be considered; electron-electron collisions, electron-optical phonon interactions, and phonon-phonon interactions. Electrons lose energy to optical mode phonons, but these have a low group velocity and do not contribute much to thermal conduction. The optical mode phonons exchange energy with the lower 
frequency acoustic mode phonons and these contribute to thermal conduction. Over long timescales these different models reduce to Fourier transport.

As with longer pulse interactions, some of the most interesting physics is to be found in phase transitions. Siwick (2003) has performed time-resolved electron diffraction experiments to demonstrate that very thin aluminium films undergo thermal melting within 3.5 ps. Siwick discusses some of the earlier work that led to the view that melting occurred within $500 \mathrm{fs}$, which is faster than the transfer of energy to the lattice. This led to the view that melting is non-thermal; the cohesive forces between the atoms are essentially reduced by the formation of the electron hole plasma. However, Siwick shows through an analysis of time-dependent diffraction data that even though the solid is highly disordered after $1.5 \mathrm{ps}$ it is nonetheless still a solid. The disorder is due to very large amplitude oscillations caused by superheating of the lattice to temperatures well above the equilibrium melting point and the material does not become fully liquid until after 3.5 ps when, in Siwick's words, "The solid shakes itself apart".

This mechanism of melting is quite different from the mechanisms operating at longer timescales, where evidence for a picture of vacancy mediated melting is beginning to emerge. The selective evaporation of volatile components of compounds semiconductors has long been recognised (Sands \& Howari, 2005) but the role of vacancies in the formation of amorphous silicon is beginning to be recognized (Lulli et al, 2006). The corresponding generation of such defects during excimer laser processing has also been observed, both after melting and solidification (La Magna et al, 2007) and immediately prior to the melting of crystalline silicon (Maekawa \& Kawasuso, 2009). These vacancies are generated primarily at the surface and melting is initiated there. At the timescales involved in ultra fast melting, however, there is no evidence as yet that vacancies are involved. Indeed, the evidence of Siwick et al points to superheating of the lattice and the homogeneous nucleation of liquid droplets within the material (Rethfield at el, 2002a). The melting time is determined in this model by the electron-phonon relaxation time.

\section{Conclusion}

Pulsed laser heating of mainly metals and semiconductors has been discussed within the framework of Fourier's law of heat conduction. A number of analytical solutions of the 1dimensional heat conduction equations have been considered. In the pre-melting regime these include the simple semi-infinite solid with surface absorption as well as a two-layer model, and analytical models of melting have also been examined. However, analytical models are limited and numerical methods of solving the heat diffusion equation have been discussed. In particular, it has been shown that Fourier's law is not well defined for abrupt changes in material properties and that the effective thermal conductivity across the interface is given by a combination of the two different conductivities. The usual parabolic form of the heat diffusion equation can give rise to errors in such circumstances, although it can be used when the spatial variations in the thermal conductivity are small, almost linear. Analytical models, such as El-Adawi's two-layer model, do not suffer this difficulty as the parabolic heat diffusion equation is usually solved on either side of the junction.

Of particular interest is the formulation of melting within numerical models. Classical thermodynamic models of melting and solidification have been discussed and shown to be found wanting, especially in relation to a diffuse interface. In this regard, phase field models 
have been discussed, but these are not well suited to 1-D heat conduction and other approaches would appear to be needed. However, a completely satisfactory and consistent numerical model of rapid heat conduction, melting and solidification has yet to be formulated, though progress has been made in this direction. For ultra-fast heating, it has been shown that heat conduction requires consideration of separate electron and phonon temperatures as well as an interaction between the two. The experimental evidence on ultrafast melting suggests that the process is thermal, being limited by the electron-phonon interaction, but models of heat conduction which incorporate melting have not been discussed. It does not seem likely at the present time that such models of melting will have much impact on models of melting on nanosecond or longer timescales as the two processes appear to be very different. On the other hand, mechanisms of solidification, which have not been discussed for ultra-fast heating, might have more in common, particularly with regard to the evolution of microstructure.

In the author's view, the problems of melting, and the associated issues of thermal conduction, constitute some of the most promising and fruitful areas of research in this field. A great deal of work remains to be done, not only in experimental studies to gather data and to characterize these processes, but also in the development of theoretical and computational models.

\section{References}

Abd El-Ghany, S. E. -S. (2001) . The temperature profile in the molten layer of a semi-infinite target induced by irradiation using a pulsed laser, Optics and Laser Technology, Vol. 33 , No. 8, (November, 2001), pp. (539-551), ISSN 0030-3992

von Allmen, Martin \& Blatter, Andreas. (1995). Laser Beam Interactions with Materials: physical principles and applications, Springer, ISBN 3-540-59401-9, Berlin.

Bechtel, J. H. (1975). Heating of solid targets with laser pulses, Journal of Applied Physics, Vol. 46, No.4 , (April, 1975), pp. (1585-1593), ISSN: 0021-8979

Cahoon, J. R (2003); On the atomistic theory of solidification, Metallurgical and Materials Transactions A Vol. 34, No. 11, (November, 2003) pp. (2683-2688), ISSN: 1073-5623

Chen, J.K., Tzou, D. Y., Beraun, J. E. (2006). A semiclassical two-temperature model for ultrafast laser heating, International Journal Of Heat And Mass Transfer, Vol. 49, No. 1-2 (January, 2006), pp. (307-316) , ISSN: 0017-9310

Dowden, J. (Ed.). (2009). The Theory of Laser Materials Processing, Springer Series in Materials Science 119, Springer , ISBN 978-1-4020-9339-5, Dordrecht, Netherlands

El-Adawi, M. K. (1986). Laser Melting of Solids - An exact solution for time intervals less or equal to the transit time, Journal of Applied Physics, Vol 60, No. 7, (October,1986), pp 2256-2259, ISSN: 0021-8979

El-Adawi, M. K. \& Shalaby, S. A. (1986). Laser Melting of Solids - An exact solution for time intervals greater than the transit time, Journal of Applied Physics, Vol. 60, No. 7, (October,1986), pp. (2260-2265), ISSN: 0021-8979

El-Adawi, M. K., Abdel-Naby, M. A. and Shalaby, S. A. (1995). Laser heating of a two-layer system with constant surface absorption: an exact solution, International Journal of Heat and Mass Transfer, Vol. 38, No. 5, (March, 1995), pp. (947-952), ISSN 0017-9310

El-Nicklawy, M.M., Hassan, A. F., -S Abd El-Ghany, S.E. (2000). On melting a semi-infinite target using a a pulsed laser, Optics and Laser Technology, Vol. 32, No. 3, (April, 2000), pp. (157-164), ISSN 0030-3992 
Howari, H., Sands, D., Nicholls, J. E., Hogg, J. H. C., Hagston, W. E., Stirner, T. (2000). Excimer laser induced diffusion in magnetic semiconductor quantum wells. Journal of Applied Physics, Vol. 88 , No. 3, (August, 2000) pp. (1373-1379), ISSN: 0021-8979

Jackson, K. A. and Chalmers, B. (1956). Kinetics of Solidification, Canadian Journal of Physics, Vol. 34, No. 5, (May, 1956) pp. (473-490), ISSN 0008-4204

Kim, S. G., and Kim, W. T., (2001). Phase-field modeling of rapid solidification, Materials Science and Engineering A, Vol. 304, Sp. Iss. SI (May, 2001) pp. (281-286), ISSN: 09215093

La Magna, A., Alippi, P., Privitera, V., Fortunato, G., Camalleri, M., Svensson, B. (2004). A phase-field approach to the simulation of the excimer laser annealing process in $\mathrm{Si}$, Journal Of Applied Physics Vol.95, No. 9, (May, 2004), pp. (4806-4814), ISSN: 00218979

La Magna A., Privitera V., Mannino G. (2007). Defect generation and evolution in laser processing of $\mathrm{Si}$, in 15th IEEE International Conference On Advanced Thermal Processing Of Semiconductors - RTP 2007, pp.(245-250), IEEE, New York, ISBN: 978-14244-1227-3

Lee, S. H. (2005). Nonequilibrium heat transfer characteristics during ultrafast pulse laser heating of a silicon microstructure, Journal of Mechanical Science And Technology Vol. 19 , No. 6, (June, 2005), pp.(1378-1389) ISSN: 1738-494X

Lowndes, D. H. , (1984). Pulsed Beam Processing of Gallium Arsenide, in Pulsed Laser Processing of Semiconductors, Semiconductors and Semimetals, Volume 23, Wood, R.F., White C.W., Young, R. T. , pp. (471-553), Academic Press, Orlando

Lulli G., Albertazzi E., Balboni S., Colombo L. (2006). Defect-induced homogeneous amorphization of silicon: the role of defect structure and population, Journal of Physics: Condensed Matter, Vol 18, No 6 (February, 2006) pp. (2077-2088) ISSN: 09538984

Maekawa M. \& Kawasuso A. (2009). Vacancy Generation in Si During Solid-Liquid Transition Observed by Positron Annihilation Spectroscopy, Japanese Journal of Applied Physics Vol. 48, No. 3, (March 2009), Art. No. 030203, ISSN: 0021-4922

Medvedev N., Rethfeld B. (2010). A comprehensive model for the ultrashort visible light irradiation of semiconductors, Journal Of Applied Physics Vol. 108 No. 10 (November, 2010 ), Art. Num. 103112 , ISSN: 0021-8979

Pusztai, T., Tegze, G., Tóth, G. I., Környei, L., Bansel, G., Fan, Z. Y., Gránásy, L. (2008). Phase-field approach to polycrystalline solidification including heterogeneous and homogeneous nucleation, Journal of Physics: Condensed Matter Vol. 20, No. 40 (October, 2008) 404205, ISSN: 0953-8984

Qin, R. S. and Bhadeshia, H. K. (2010). Phase field method, Materials Science and Technology, Vol. 26 No. 7 (July, 2010) pp. (803-811), ISSN 0267-0836

Rethfeld B., Sokolowski-Tinten K., von der Linde D. (2002a). Ultrafast thermal melting of laser-excited solids by homogeneous nucleation, Physical Review B, Vol. 65 No. 9 , (March 2002), Art. No. 092103, ISSN: 1098-0121

Rethfeld B., Kaiser A., Vicanek M., Simon G. (2002b). Ultrafast dynamics of nonequilibrium electrons in metals under femtosecond laser irradiation, Physical Review B, Vol. 65, No. 21 (June, 2002) Art. No. 214303 , ISSN: 1098-0121 
Sands, D., Key, P. H., Hoyland, J. D., (1999). In-situ measurements of excimer laser irradiated zinc sulphide films on silicon, Applied Surface Science, Vol. 138, Nos. 1-2, (January, 1999) pp. (240-243), ISSN 0169-4332

Sands, D., Howari, H. (2005). The kinetics of point defects in low-power pulsed laser annealing of ion-implanted $\mathrm{CdTe} / \mathrm{CdMnTe}$ double quantum well structures, $J$ Journal of Applied Physics Vol. 98, No. 8, (October, 2005), Art. No. 083506, ISSN: 00218979

Sands, D. (2007). New theory of undercooling during rapid solidification: application to pulsed laser heated silicon, Applied Physics A-Materials Science E Processing, Vol. 88, No. 1, (July, 2007), pp. (179-189), ISSN: 0947-8396

Sekerka, Robert F. (2004). Morphology, from sharp interface to phase field models, Journal of Crystal Growth, Vol. 264, No.4 (March, 2004) pp. (530-540), ISSN 0022-0248

Shahzade, Z., Shuja, Y., Bekir S. and Momin, O. (2010). Laser Heating of Moving Solid: Influence of Workpiece Speed on Melt Size, AIChE (American Institute of Chemical Engineers) Journal Vol. 56, No. 11 (November, 2010) pp (2997-3004)

Shih, C. J., Fang, C. H. , Lu, C. C. , Wang, M. H., Lee, M. H. and Lana, C. W. (2006). Phase field modeling of excimer laser crystallization of thin silicon films on amorphous substrates, Journal Of Applied Physics,Vol. 100, No. 5, (September 2006) art. 053504, ISSN: 0021-8979

Shneidman, Vitaly A. (1995). Theory of time-dependent nucleation and growth during a rapid quench, Journal of Chemical Physics, Vol. 103, No. 22, (December, 1995), pp. ( 9772-9781), ISSN 0021-9606

Shneidman, Vitaly A. (1996). Interplay of latent heat and time-dependent nucleation effects following pulsed-laser melting of a thin silicon film, Journal of Applied Physics, Vol. 80, No. 2, (July, 1996), pp. (803-811), ISSN: 0021-8979

Shneidman, Vitaly A. \& Weinberg, Michael C. (1996). Crystallization of rapidly heated amorphous solids, Journal of Non-Crystalline Solids, Vol. 194, Nos. 1-2, (January, 1996) pp. (145-154), ISSN: 0022-3093

Shuja, S. Z., Yilbas, B. S. and Momin, O. (2011). Laser repetitive pulse heating and melt pool formation at the surface, Journal of Mechanical Science and Technology, Vol. 25, No. 2 (February, 2011) pp. (479-487), ISSN: 1738-494X

Siwick, B.J., Dwyer, J. R., Jordan, R. E., Miller, R. J. D. (2003). An atomic-level view of melting using femtosecond electron diffraction, Science, Vol. 302, No 5649, (November, 2003) pp. (1382-1385), ISSN: 0036-8075

Silva, J. B. C., Romão, E. C. , de Moura, L. F. M. (2008). A comparison of time discretization methods in the solution of a parabolic equation, $7^{\text {th }}$ Brazilian Conference on Dynamics, Control and Applications, May07-09, 2008, Retrieved from

$<\quad$ http://www4.fct.unesp.br/ dmec/dincon2008/artigos/02/01\%20-\%2002Campos_Silva.pdf $>$

Smith, G. D. (1965). Numerical Solution of Partial Differential Equations, Oxford University Press, London

Sonkusare, A., Sands, D., Rybchenko, S. I., Itskevich, I. (2005). Photoluminescence from Ion Implanted and Low-Power-Laser Annealed GaAs/AlGaAs Quantum Wells, AIP Conference Proceedings Vol. 772, (June, 2005), pp. (965-966), PHYSICS OF SEMICONDUCTORS - 27th International Conference on the Physics of Semiconductors (ICPS 27) 
Steinbach, I., and Apel, M. (2007). Phase-field simulation of rapid crystallization of silicon on substrate, Materials Science and Engineering A, Vol. 449 (March, 2007) pp. (95-98), ISSN: 0921-5093

Sung, Y.H., Takeya, H., Hirata, K., Togano, K. (2003). Specific heat capacity and hemispherical total emissivity of liquid $\mathrm{Si}$ measured in electrostatic levitation, Applied Physics Letters Vol. 83, No. 6, (August 2003), pp. (1122-1124), ISSN: 00036951

Wood R. F. and Jellison, G. E., Jr., (1984). Melting Model of Pulsed Laser processing, in Pulsed Laser Processing of Semiconductors, Semiconductors and Semimetals, Volume 23, Wood, R.F., White C.W., Young, R. T. , pp. (165-250), Academic Press, Orlando.

Wu, David T. (1997), Nucleation Theory, Solid State Physics, volume 50, H. Ehrenreich and F. Spaepen, eds. pp. 37-187, Academic Press, San Diego

Xie, J. \& Kar, A. (1997). Mathematical modelling of melting during laser materials processing, Journal of Applied Physics, Vol. 81, No. 7 pp. (3015-3022), ISSN: 0021-8979

Yilbas, B. S., Shuja, S. Z. (1999). Laser short-pulse heating of surfaces, Journal of Physics D: Applied Physics, Vol. 32 No. 16, (August, 1999), pp. (1947-1954), ISSN: 0022-3727

Yilbas, B. S., Shuja, S. Z. (2000). Electron kinetic theory approach for sub-nanosecond laser pulse heating, Proceedings of the Institution of Mechanical Engineers, Part C: Journal of Mechanical Engineering Science, Vol. 214 No. 10, (2000) pp. (1273-1284), ISSN: 09544062 


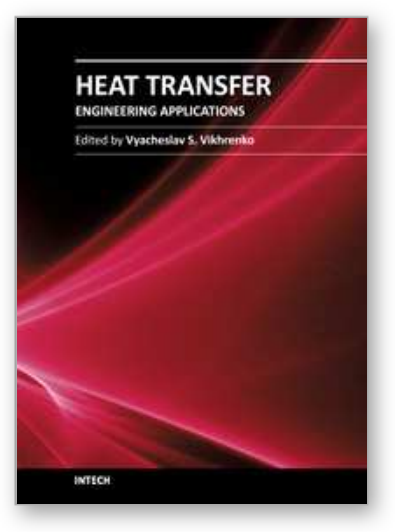

\author{
Heat Transfer - Engineering Applications \\ Edited by Prof. Vyacheslav Vikhrenko
}

ISBN 978-953-307-361-3

Hard cover, 400 pages

Publisher InTech

Published online 22, December, 2011

Published in print edition December, 2011

Heat transfer is involved in numerous industrial technologies. This interdisciplinary book comprises 16 chapters dealing with combined action of heat transfer and concomitant processes. Five chapters of its first section discuss heat effects due to laser, ion and plasma-solid interaction. In eight chapters of the second section engineering applications of heat conduction equations to the curing reaction kinetics in manufacturing process, their combination with mass transport or ohmic and dielectric losses, heat conduction in metallic porous media and power cables are considered. Analysis of the safety of mine hoist under influence of heat produced by mechanical friction, heat transfer in boilers and internal combustion engine chambers, management for ultrahigh strength steel manufacturing are described in this section as well. Three chapters of the last third section are devoted to air cooling of electronic devices.

\title{
How to reference
}

In order to correctly reference this scholarly work, feel free to copy and paste the following:

David Sands (2011). Pulsed Laser Heating and Melting, Heat Transfer - Engineering Applications, Prof. Vyacheslav Vikhrenko (Ed.), ISBN: 978-953-307-361-3, InTech, Available from: http://www.intechopen.com/books/heat-transfer-engineering-applications/pulsed-laser-heating-and-melting

\section{INTECH}

open science | open minds

\section{InTech Europe}

University Campus STeP Ri Slavka Krautzeka 83/A

51000 Rijeka, Croatia Phone: +385 (51) 770447

Fax: +385 (51) 686166

www.intechopen.com

\section{InTech China}

Unit 405, Office Block, Hotel Equatorial Shanghai

No.65, Yan An Road (West), Shanghai, 200040, China

中国上海市延安西路65号上海国际贵都大饭店办公楼405单元

Phone: +86-21-62489820

Fax: +86-21-62489821 
(C) 2011 The Author(s). Licensee IntechOpen. This is an open access article distributed under the terms of the Creative Commons Attribution 3.0 License, which permits unrestricted use, distribution, and reproduction in any medium, provided the original work is properly cited. 